\title{
An Introduction to the World Space Observatory-Ultraviolet Spectrographs
}

\author{
S. Hermanutz ${ }^{1}$, J. Barnstedt ${ }^{1}$, S. Diebold ${ }^{1}$, H.R. Elsener ${ }^{2}$, P.R. Ganz ${ }^{3}$, C. Kalkuhl ${ }^{1}$, \\ N. Kappelmann ${ }^{1}$, M. Pfeifer ${ }^{1}$, O. Tanirah ${ }^{3}$, M. Sachkov ${ }^{4}$, D.M. Schaadt ${ }^{3,5}$, T. Schanz $^{1}$, \\ B.M. Shustov 4 , K. Werner ${ }^{1}$ \\ ${ }^{1}$ Institute for Astronomy and Astrophysics (IAAT), Eberhard Karls University Tübingen \\ ${ }^{2}$ Empa, Swiss Federal Laboratories for Materials Science and Technology, Dübendorf \\ ${ }^{3}$ Karlsruhe Institute of Technology (KIT), DFG-Center for Functional Nanostructures \\ 4 Institute of Astronomy of the Russian Academy of Sciences \\ ${ }^{5}$ Institute of Energy Research and Physical Technologies, Clausthal University of Technology
}

\begin{abstract}
The World Space Observatory Ultraviolet (WSO-UV) is a multinational mission under the leadership of Russia with contributions of Spain and Germany. The mission is part of the Spektrum series and launch is currently scheduled for 2016. It consists of a $1.7 \mathrm{~m}$ mirror focusing on spectrographs in the range of $102-310 \mathrm{~nm}$ with a resolution of $\mathrm{R} \geq 55,000$ for high resolution spectral observations, a long-slit-spectrograph for spatially resolved observations and an imager. According to the Phase-B-Study all spectrographs will use the same detectors built by the IAAT. These spectrographs are designed to observe cosmic plasma with temperatures of several ten thousands Kelvin and atomic transition lines of all important atoms and molecules like $\mathrm{H}_{2}, \mathrm{CO}, \mathrm{OH}$ etc. In the core program the community wants to determine the amount of baryonic matter in the universe, extend the knowledge about the formation of galaxies and analyze the atmospheres of extrasolar planets and protoplanetary discs. To achieve these goals the IAAT designed in cooperation with the Leibniz-Institute for Analytical Sciences (ISAS Berlin) the spectrographs. In addition Tübingen develops and builds a new type of microchannel plate detector based on gallium nitride cathodes and a cross-strip-anode.
\end{abstract}

Keywords: WSO-UV, WUV spectrographs, gallium nitride, micro channel plate detector

\section{THE WORLD SPACE OBSERVATORY - UV}

The probable shutdown of the Hubble Space Telescope (HST) at around 2015 will end two and a half productive decades of UV astronomy and there is no other ESA or NASA mission planned to replace HST. WSO-UV as a multinational project under leadership of Russia will offer a new perspective on the UV universe with a spectral resolution of more than 55,000 in the spectral range of 102-310 nm. The major participants of this mission are Russia, responsible for the spacecraft, spectrographs, launch and the satellite bus, Spain, which contributes an imaging instrument and participates in the ground control for the satellite. The Institute for Astronomy and Astrophysics Tübingen (IAAT) was involved in the design of the spectrographs and is developing the microchannel plate (MCP) detectors for the high resolution spectrographs. The mission launch is scheduled for 2016 and observations should last for five years with the possibility of a five years extension.

\subsection{Satellite Bus}

WSO-UV project ${ }^{1,2}$ will use the Russian NAVIGATOR platform which was designed by Lavochkin Science \& Technology Association as a unified bus for several missions including the Russian meteorological mission Elektro and the Spektrum series of the Russian space program consisting of Radioastron, Spektrum-Roentgen-Gamma (launch scheduled 2013) and WSO-UV. The first two launches of the platform (with ELECTRO and Radioastron satellites in 2011) has demonstrated its good performance capacity. WSO-UV will be launched from Baikonur (Kazakhstan) with a Zenit-2 rocket and a Fregat-SB booster. The platforms weighs $1300 \mathrm{~kg}$ and has a payload

hermanutz@astro.uni-tuebingen.de

Space Telescopes and Instrumentation 2012: Ultraviolet to Gamma Ray,

edited by Tadayuki Takahashi, Stephen S. Murray, Jan-Willem A. den Herder,

Proc. of SPIE Vol. 8443, 844304 - @ 2012 SPIE · CCC code: 0277-786/12/\$18 - doi: 10.1117/12.925147

Proc. of SPIE Vol. $8443844304-1$ 


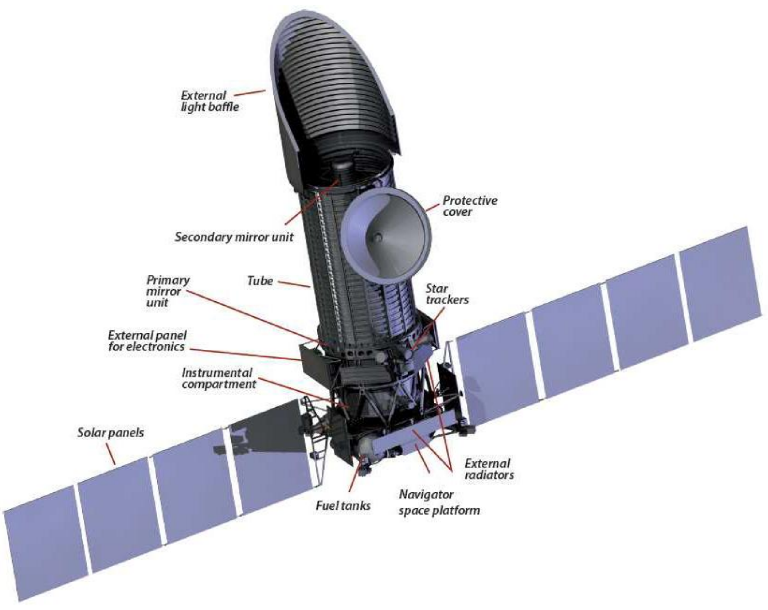

Figure 1. Schematic of the NAVIGATOR platform and WSO-UV

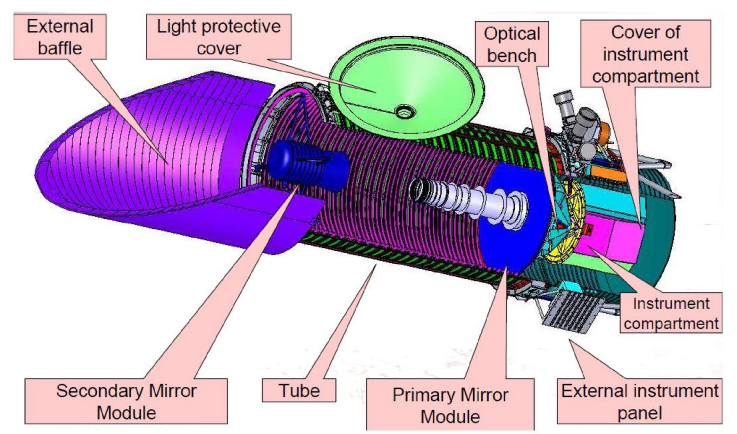

Figure 2. Schematic of the Telescope of the WSO-UV

mass of $1600 \mathrm{~kg}$. With assistance through the fine guidance system the pointing stabilization is $\leq 0.1$ arcseconds. The bus provides $300 \mathrm{~W}$ power for all instruments and a data download up to $4 \mathrm{Mbit} / \mathrm{s}$ in the case of WSO-UV. The WSO-UV telescope will have a $1.7 \mathrm{~m}$ mirror and a Ritchey-Chretien optics with a focal length of $17 \mathrm{~m}$ and a field of view of 30 arcseconds (see Figs. 1 and 2).

\subsection{Instrumentation}

There will be two main instruments on board of WSO-UV: the WSO-UV Spectrographs (WUVS) and the Imaging and Slitless Spectroscopy Instrument for Surveys (ISSIS). The WUVS consist of three parts:

- VUVES : Vacuum Ultraviolet Echelle Spectrograph

- UVES : Ultraviolet Echelle Spectrograph

- LSS: Long Slit Spectrograph

The first two instruments will use Echelle optics with a high spectral resolution in the wavelength ranges of $102-176 \mathrm{~nm}$ and $174-310 \mathrm{~nm}$ and the LSS is based on a Rowland design for low spectral resolution observations of extended objects with a spatial resolution of 1 arcsecond with a field of view of 75 arcseconds. For more details about ISSIS see Gomez de Castro. ${ }^{3}$

\section{THE WUV SPECTROGRAPHS}

The WUV spectrographs were designed in cooperation with the Leibniz Institute for Analytical Sciences as heritage of the Tübingen participation in the ORFEUS program from 1993-1996. Based on the ORFEUS detectors properties the design was in the past 20 years improved and integrated as instrument into the WSOUV telescope. In the following section the spectrographs which passed through multiple studies changing and optimizing their design will be presented (mainly the Phase-B-Study results).

\subsection{Scientific Goals}

The spectrograph was designed as an observatory to cover multiple fields of interest. In general, WSO-UV will not compete with but complement HST instruments. In the vacuum UV range it provides a comparable high spectral resolution and in the UV range it will have a higher effective area compared to COS and STIS. In the core program the following items should be investigated:

- Hot plasma with temperatures up to $300,000 \mathrm{~K}$ 
- The formation of the milky way

- The distribution of baryonic matter in the universe

- Accretion processes

- Atmospheres of extrasolar planets

- Protoplanetary discs next to strong UV sources

A more detailed discussion was presented in Gomez de Castro et al. ${ }^{4}$

\subsection{Design of the WUV Spectrographs}

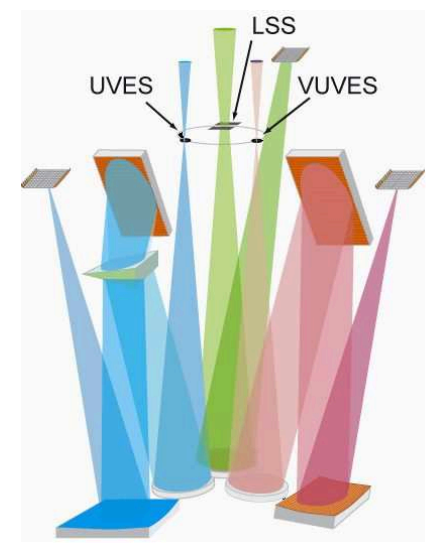

Figure 3. Schematic of the optical design ${ }^{5}$

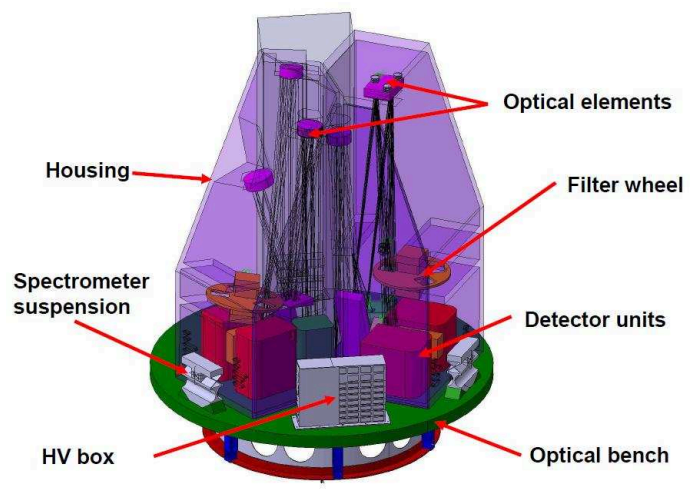

Figure 4. Integration of the instrument into the telescope as planed in a Phase-B-Study ${ }^{5}$

The design of the spectrographs takes into account, that every UV instrument needs as few optical elements as possible to reduce the loss of photons due to the lack of good reflective coatings for the UV range. Beside good coatings for the optical elements and a small angle incidence, a solar blind detector is required to reduce the amount of stray background light which determines the lowest observable brightness of the sources. These requirements led to the optical design shown in Fig. 3. The VUV spectrograph comprises a collimator to align the beam on the Echelle grating and a camera mirror including the cross disperser grating to focus on the detector. The UV spectrograph uses the same optical design but instead of a grating a prism is used for the cross dispersion, due to its higher efficiency in the range of $174-310 \mathrm{~nm}$. The long slit spectrograph uses a Rowland design to focus the incoming light on two separated detectors. In the LSS the same detectors as in VUVES and UVES will be implemented. Within multiple studies carried out by the German space industry different structural designs were compared. In Fig. 4 each spectrograph has its own optical bench therefore each instrument can be manufactured, tested and integrated on its own. The last design uses only one optical bench for all three spectrographs to avoid higher mass and alignment efforts. There were three suspensions foreseen to establish the isostatic mounting of the WUVS optical bench to the optical bench of the telescope. In the first designs additional focusing mechanics were foreseen to correct for thermal instabilities of the optical bench but in the Phase-B-Study a new material $\mathrm{CeSiC}$ (carbon-fiber reinforced silicon carbide $^{6}$ ) was investigated. In comparison to standard materials for the optical bench like aluminum or titan which allow thermal variabilities of $\pm 1.5^{\circ} \mathrm{C} \mathrm{CeSiC}$ provides stability within a thermal range of $\pm 4^{\circ} \mathrm{C}$, which solves most of the heat based problems. In the Phase-B-Study a prototype of the whole optical bench was manufactured and fulfilled all thermal requirements but showed structural deficits due to fissures. This material is still very promising but needs further investigation on manufacturer side to get a TRL $\geq 5$. The design also includes a fine guidance system which is not placed on the main optical bench but on the instruments bench next to the light entrance slits to minimize the distance and optimize the performance of the system. As the point spread function of the main mirror is smaller than the aperture, a slit monitoring system, to ensure that the observed objects is fully within the aperture, was integrated. These studies also have foreseen redundant detectors for the Echelle spectrographs to improve the lifetime. A mirror and filter system is foreseen between the main and redundant detectors heads to a allow the observation of brighter sources. 


\subsection{Simulated Performance of the WUV Spectrographs}

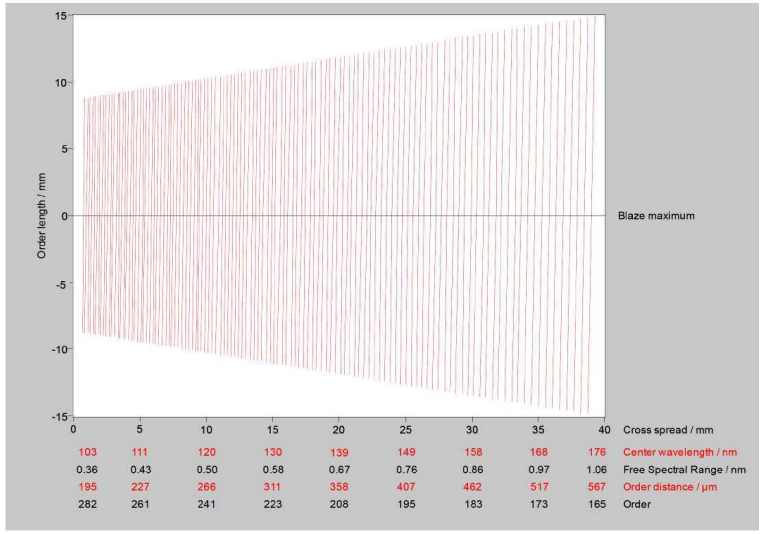

Figure 5. Simulated location of VUV Echelle rders on the detector

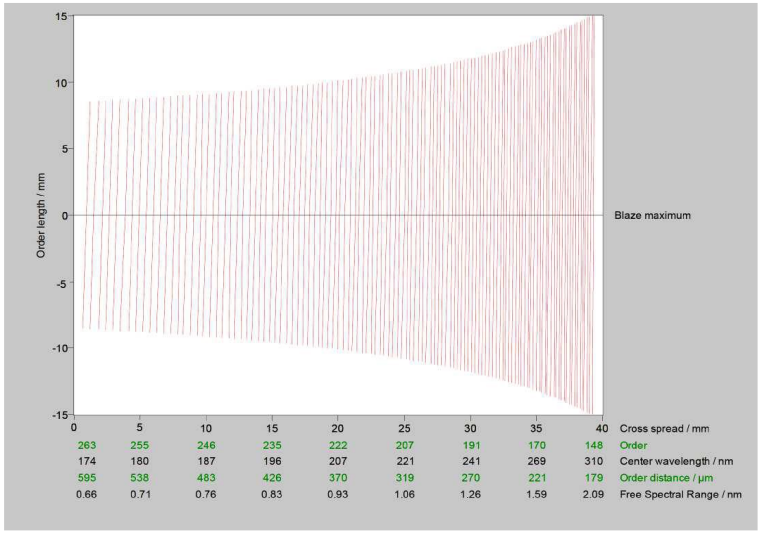

Figure 6. Simulated location of UV Echelle orders on the detector

Comparison of effective areas

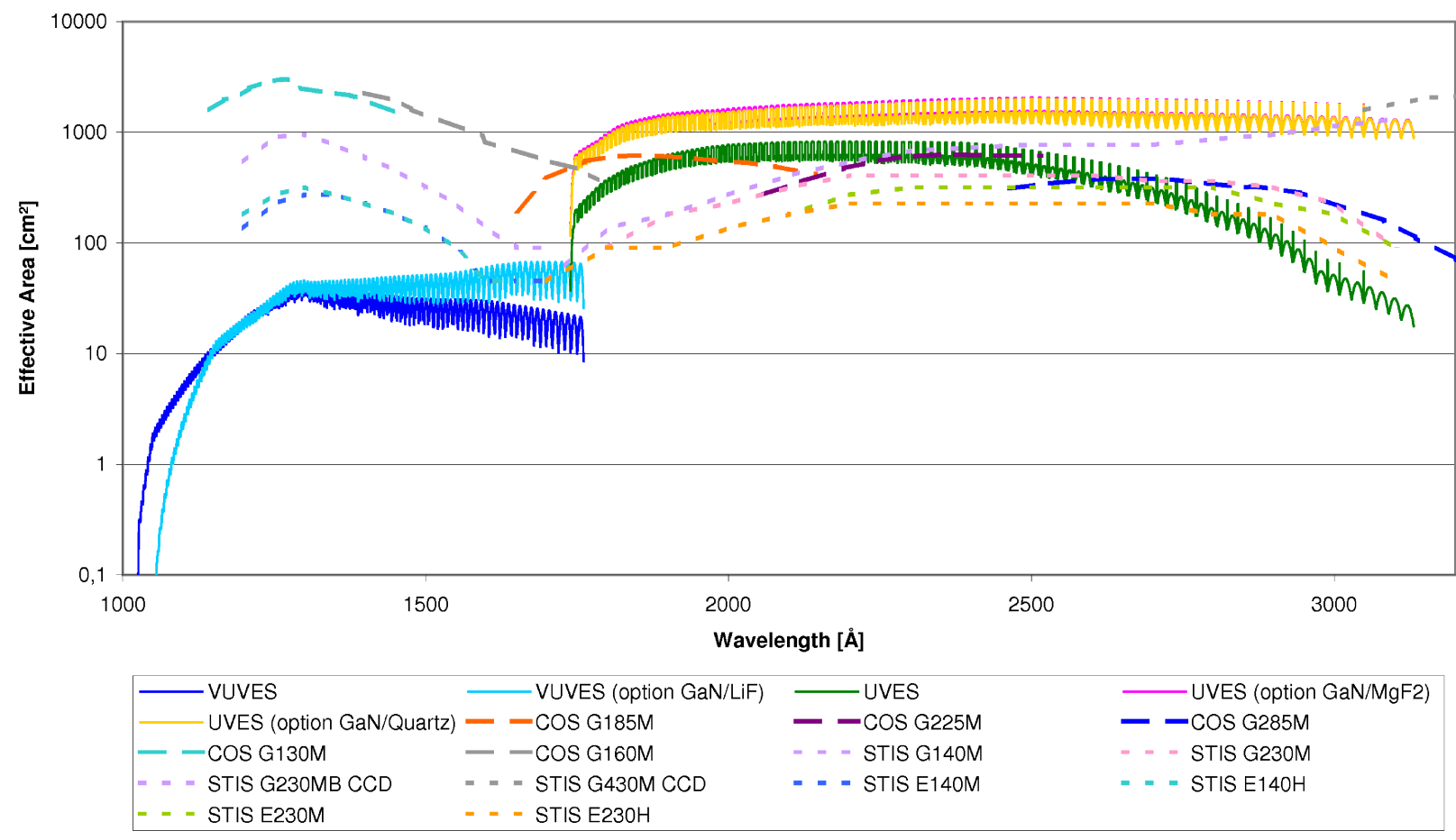

Figure 7. Comparison of preliminary simulated UVES and VUVES effective areas with those of HST instruments

The two main spectrographs VUVES and UVES cover the ranges from 102-176 nm and 174-310 nm. The lower cutoff was set to include the possibility to detect the important O VI resonance line transition at $103 \mathrm{~nm}$. The combination of an optical resolution of $80 \mu \mathrm{m}$ and a pixel size of $30 \mu \mathrm{m}$ grants, according to the Phase-B-Study, for VUVES a spectral resolution of around 55,000 and for UVES a spectral resolution of around 50,000. With the use of a slit monitoring system which determines the exact position of the object within the aperture, the resolution could be improved by approximately $20 \%-30 \%$ by correcting for the telescope jitter. In Figs. 5 and 6 
the simulated location of the Echelle orders on the detector is shown with a minimum order distance of $180 \mu \mathrm{m}$. The prism which is used in the UVES design leads to the curves shape of the order distribution. In Fig. 7 the individually calculated effective areas for different cathode materials are compared with the HST instruments. For the cathodes either CsI and CsTe as standard coatings for VUVES and UVES or GaN as development item (quantum efficiency see ${ }^{7}$ ) were simulated. For the mirror reflectivity $(20 \%$ at $105 \mathrm{~nm}, 60 \%$ at $130 \mathrm{~nm}$ and more than $75 \%$ for 190-600 nm) and gratings efficiency (VUVS Echelle grating efficiency $15 \%$ at $103 \mathrm{~nm}, 17 \%$ at $139 \mathrm{~nm}, 19 \%$ at $175 \mathrm{~nm}$ and about $60 \%$ for the UVS Echelle gratings) conservative assumptions were made. In the UVES range the design exceeds the HST instruments in effective area, in the VUVES range the effective area and spectral resolution of the HST instruments is still higher, but the WUVS only need a single exposure to cover their whole wavelength range, which is also greater, whereas the HST instruments need more than one exposure with their high resolution gratings E140H (three exposures) and E230H (six exposures).

\section{THE MCP DETECTORS FOR THE WUV SPECTROGRAPHS}

Based on flight hardware experiences IAAT is developing the MCP detectors for the WUV spectrographs. MCP detectors provide solar blindness, single photon counting capabilities and a fast readout corresponding to a short dead time of the detector electronics. Furthermore there are sundry options for the cathode material and the readout anode which can be changed and applied to spectrographs without changing their design.

\subsection{Working Principle}

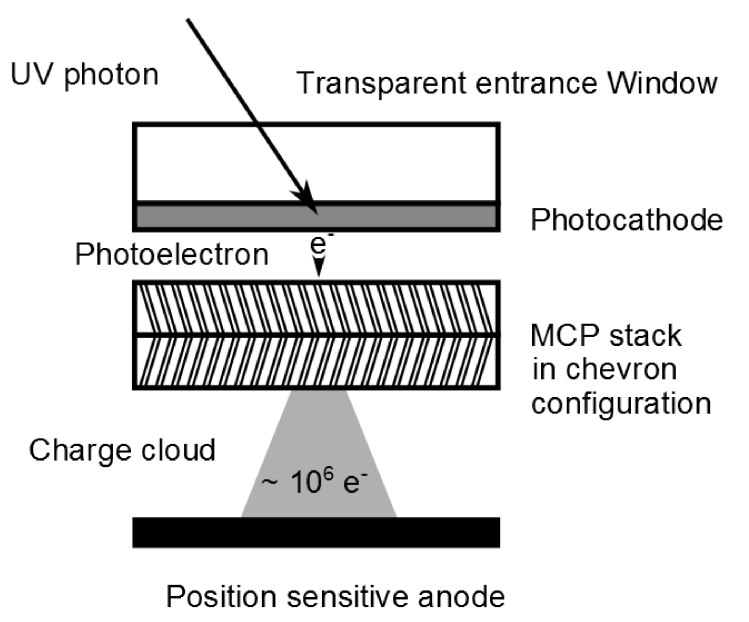

Figure 8. Detector principle in semitransparent mode ${ }^{8}$

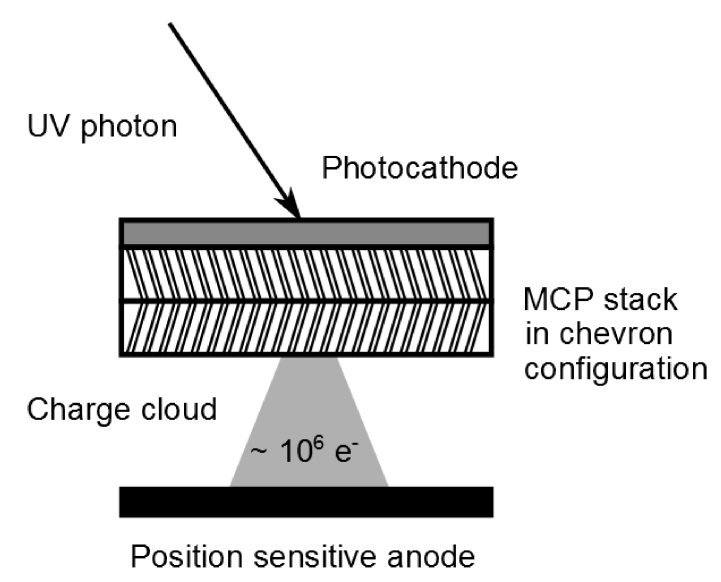

Figure 9. Detector principle in opaque mode

There are two general concepts for MCP detectors, the opaque and the semitransparent modes (shown in Figs. 8 and 9). Due to the fact, that the MCPs need a pressure below $10^{-5}$ mbar to work properly and the cathodes degrade rapidly under atmosphere exposure, the semitransparent mode uses a sealed detector tube with an entrance window which is coated with the cathode material. If the detector is used in an evacuated environment or in space this window can be abandoned and the cathode can be directly coated in opaque mode on the MCPs. Independent of the configuration an incoming photon hits the cathode material and releases an electron. This electron will be accelerated through the MCPs which amplify the incoming electron by a factor of $10^{5}$ to $10^{7}$ depending on the voltage and MCP configuration. This electron cloud hits a charge sensitive anode and the centroid of the electron cloud can be calculated. The more sensitive the anode is, the less electrons are needed for a good calculation of the centroid. As each MCP has a maximum amount of charge it can provide, the gain factor determines the MCP lifetime and therefore a more sensitive anode enhances the lifetime of the detector. A sealed tube is easier to handle but the use of an entrance window reduces, due to its specific transmission properties, the detectable wavelength range. 


\subsection{Possible Configurations for VUVES and UVES}

For both spectrographs two different detector options are under consideration. For VUVES the detector will be used in opaque mode with a shutter system, which will be opened in space. Therefore there is no lower wavelength cutoff due to an entrance window and the wavelength range of $102-176 \mathrm{~nm}$ is only limited by the telescope, optics and the quantum efficiency of the cathode material. The cathode material will be either CsI as a commonly used material or GaN. GaN shows a much higher quantum efficiency ${ }^{7}$ under laboratory conditions but was not flown in space until now. If the quantum efficiency of GaN in semitransparent mode exceeds the one of CsI in opaque mode a reduction in wavelength range has to be considered for a better sensitivity in the remaining range. For UVES there is either the possibility of using CsTe as standard material or GaN in semitransparent mode on a $\mathrm{MgF}_{2}$ window, which grants transmission down to $110 \mathrm{~nm}$, or using $\mathrm{GaN}$ in opaque mode which would possibly need a sealed detector tube, too. As all detectors have the same structural properties (size, weight and electrical connectors) except for the quantum efficiency they can be exchanged without influencing the general instrument. This allows a wide range of parallel developments and the use of the best solution without excluding any possibility in an early stage of the development.

\subsection{Gallium Nitride}

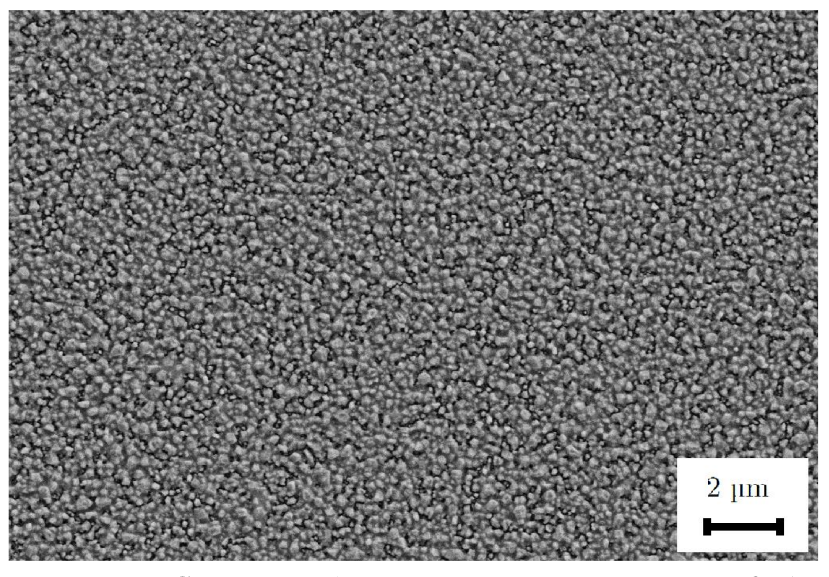

Figure 10. Scanning electron microscope picture of the grown polycrystalline GaN

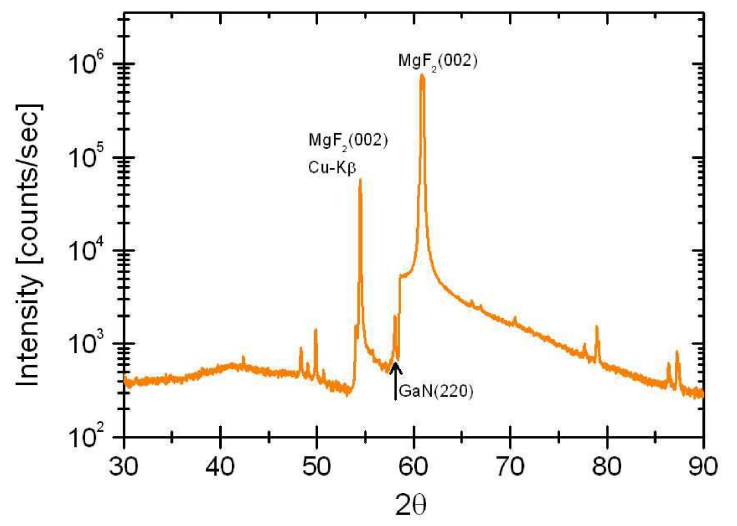

Figure 11. Powder diffractometry analysis of the grown $\mathrm{GaN}$

The GaN cathodes will be produced with molecular beam epitaxy but transferred and assembled to the detectors in Tübingen. The main challenge aside from a high quantum efficiency is a low growing temperature to coat the MCPs in opaque mode without harming them. As shown in Fig. 10 polycrystalline GaN was successfully grown at low temperatures with a high uniformity in grain coverage and size. The main peaks in the powder diffractometry analysis are clearly identified as cubic GaN. The other peaks and the slow lowering for $2 \Theta \geq 60$ are known effects of the measurement device and can be ignored. In the next step these samples will be doped with Mg to optimize the band alignment of GaN, transferred to Tübingen, activated with Cs to form a working cathode and characterized. For the characterization in the wavelength range $200-800 \mathrm{~nm}$ a monochromator system with a resolution of $0.1 \mathrm{~nm}$ will be used and for lower wavelengths a vacuum monochromator is available at IAAT.

\subsection{Readout Electronics Based on BEETLE Chip}

The constraints for the readout electronics comprise low energy consumption (less than $10 \mathrm{~W}$ ), a high resolution with at least three pixels for one optical resolution element of the Echelle spectrum and a high charge sensitivity to extend the lifetime of the MCPs. These requirements are matched best by a cross-strip anode. The cross-strip anode covers an area of $30 \times 40 \mathrm{~mm}^{2}$ with $64 \times 64$ pixels and offers a sub sampling factor of 32 to obtain a virtual 2048x2048 pixel resolution with a lower gain than comparable anodes like the time-delay anode or the wedgeand-strip anode. But in contrast to a time-delay anode the cross-strip anode needs a readout for 128 channels. To fulfill the power and space constraints the BEETLE chip, developed by Max Planck Institute Heidelberg for 


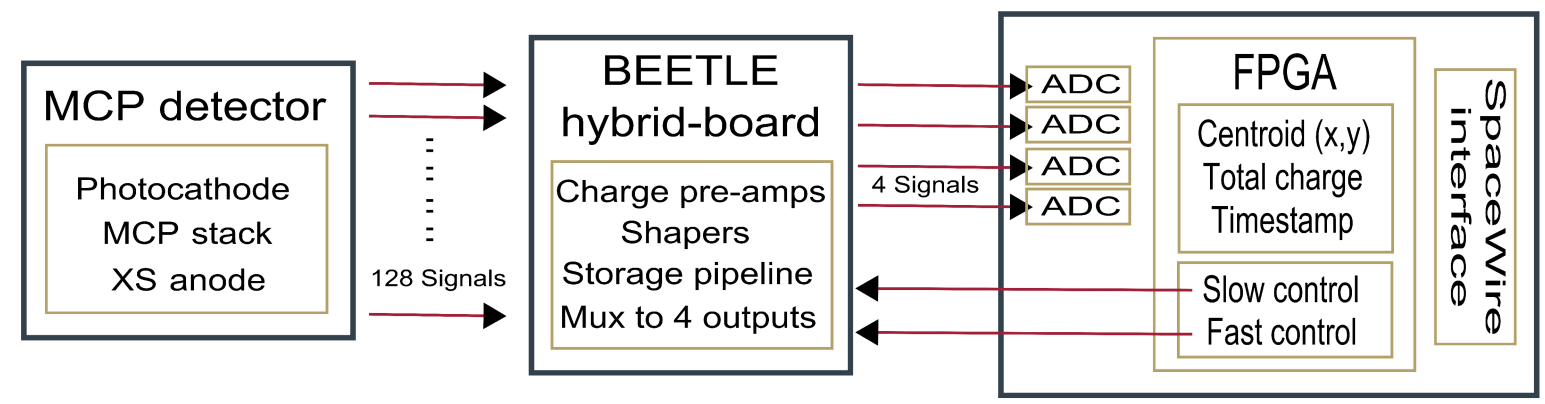

Figure 12. Schematic of the readout electronics developed at Tübingen ${ }^{9}$

the Large Hadron Collider $b$ experiment, will be used with its preamplifier and shaper properties. The internal multiplexer reduces the input to four output lines as shown in Fig. 12. The obtained data will be preprocessed in the BEETLE chip and forwarded to the FPGA control board, where the centroid is calculated. The data transfer to the ground station will use a SpaceWire interface. As fall-back option the time-delay-anode needs a higher MCP gain reducing the lifetime, but will fulfill the power constraints.

\section{SUMMARY}

The Phase-B-Study of the WSO-UV Spectrographs showed clearly that they offer a high performance and can make WSO-UV a telescope opening new options for science. The detector development in Tübingen benefits from the long-time experience with UV instruments and offers a broad range of possibilities with different modifications and fall-back options for each development item to grant an optimal instrument performance.

\section{ACKNOWLEDGMENTS}

This work is funded by Deutsches Zentrum für Luft- und Raumfahrt (DLR) grant 50QT1003.

\section{REFERENCES}

1. Shustov, B.M., Sachkov, M., Gomez de Castro, A.I., et al. "WSO-UV-ultraviolet mission for the next decade," in [Astrophysics and Space Science, Vol. 320, p. 187 - 190] (2009)

2. Shustov, B.M., Sachkov, M., Gomez de Castro, A.I., et al. "World space observatory-ultraviolet among UV missions of the coming years", in [Astrophysics and Space Science, Vol. 335, p. 273 - 282] (2011)

3. Gomez de Castro, A., Sanchez, N., Sestito, P., et al., "ISSIS: the imaging and slitless spectroscopy instrument for surveys in the World Space Observatory ultraviolet space telescope," in [SPIE Conference Series], Presented at SPIE Conference 8433 (2012).

4. Gomez de Castro, A.I., Pagano, I., Sachkov, M., et al.: In: Chavez, M., Bertone, E., Rosa-Gonzalez, D., Rodriguez-Merino, L.H. (eds.) New Quests in Stellar Astrophysics. II. Ultraviolet Properties of Evolved Stellar Populations, p. 319. Springer, Berlin (2009)

5. Werner, K., Barnstedt, J., Gringel, W., Kappelmann, N., et al., "HIRDES - The High-Resolution DoubleEchelle Spectrograph for the World Space Observatory Ultraviolet (WSO/UV)," in [Advances in Space Research vol. 41] (2008)

6. Reutlinger, A., Sachkov, M., Gal, C., Brandt, C., et al., "Using the CeSiC material for the WSO-UV spectrographs," in [Astrophysics and Space Science, Vol. 335, p. 311 - 316] (2011)

7. Siegmund, O., et al., 2008, "Gallium nitride photocathode development for imaging detectors," in [Proc. of SPIE Vol. 7021 70211B-2] (2008)

8. Diebold, S., Barnstedt, J., Kappelmann, N., Werner, K., et al., "MCP Detector Development for WSO-UV," in [SPIE Conference Series], Presented at SPIE Conference 8433 (2012)

9. Pfeifer, M., Barnstedt, J., Kappelmann, N., Werner, K., et al., "Low-power readout electronics for micro channel plate detectors with cross-strip anodes," in [SPIE Conference Series], Presented at SPIE Conference $8433(2012)$ 